\title{
Episodic memory in transient global amnesia: encoding, storage, or retrieval deficit?
}

Francis Eustache, Béatrice Desgranges, Peggy Laville, Bérengère Guillery, Catherine Lalevée, Stéphane Schaeffer, Vincent de la Sayette, Serge Iglesias, Jean-Claude Baron, Fausto Viader

\begin{abstract}
Objectives-To assess episodic memory (especially anterograde amnesia) during the acute phase of transient global amnesia to differentiate an encoding, a storage, or a retrieval deficit.

Methods-In three patients, whose amnestic episode fulfilled all current criteria for transient global amnesia, a neuropsychological protocol was administered which included a word learning task derived from the Grober and Buschke's procedure.

Results-In one patient, the results suggested an encoding deficit, and in two others, a storage deficit.

Conclusions-The encoding/storage impairment concerning anterograde amnesia documented in our patients stands in clear contrast with the impairment in retrieval which must underly the retrograde amnesia that also characterises transient global amnesia. This dissociation in turn favours the idea of a functional independence among the cognitive mechanisms that subserve episodic memory.

(F Neurol Neurosurg Psychiatry 1999;66:148-154)
\end{abstract}

Keywords: encoding; storage; retrieval; anterograde amnesia; retrograde amnesia; episodic memory

INSERM U320 and

Services de

Neurologie, CHU Côte

de Nane, 14033 Caen

Cedex, France

F Eustache

B Desgranges

P Laville

B Guillery

C Lalevée

S Schaeffer

$\mathrm{V}$ de la Sayette

$S$ Iglesias

F Viader

INSERM U320, Centre Cycéron, Boulevard Becquerel, BP 5229, 14074 Caen, France J-C Baron

Correspondence to: Professor Francis Eustache, INSERM U 320, Services de Neurologie, CHU Côte de

Nacre, 14033 Caen Cedex,

France.

Received 30 December 1997 and in final form

14 July 1998

Accepted 3 August 1998 evertheless this division into processing stages continues to be useful in helping understand the working of memory systems". These three stages may be defined in the following way: (1) encoding, during which perceptive information is transformed into more or less stable mental representations; (2) storage (or consolidation), during which mnemonic information is associated with other representations and maintained in long term memory; (3) retrieval, during which the subject can momentarily reactivate mnemonic representations. These definitions will be used in the present study.

Regarding the retrograde amnesia of TGA, it should depend on a problem of retrieval of information from episodic memory, ${ }^{3}$ as suggested by (1) the sudden nature of the episode (which excludes, for example, a progressive anterograde amnesia); (2) the fact that amnesia can affect very distant events (even if patchy ${ }^{11}{ }^{12}$ ); and (3) its transient nature-that is, the prior memories becoming available again after the attack (with the exception of a "blank" covering the acute phase and possibly the few preceding hours).

Contrary to retrograde amnesia, however, the mechanisms responsible for the anterograde amnesia have not been elucidated. Although some have suggested that they may differ from those involved in retrograde amnesia and have considered a consolidation disorder, experimental support was not provided. ${ }^{3}{ }^{10}{ }^{15} 16$ Other authors argued that the memory disturbance was the result of a deficit either in encoding ${ }^{4}$ or in retrieval. ${ }^{17} \mathrm{~A}$ systematic study of these processes should afford a better understanding of the clinical characteristics of this syndrome. Furthermore, to quote Caffara et al, ${ }^{16}$ TGA represents an "interesting "experimentum naturae' on the mechanisms of memory", both at the cognitive and at the neurobiological level. ${ }^{18}$ Transient global amnesia offers a unique opportunity to study dysfunctioning human memory in the absence of the reorganisation phenomena which take place in permanent amnesic syndromes. In addition, the patient can act as his own control, a feature of particular interest in an area such as memory, which is characterised, even in the normal subject, by a large intersubject variability.

The principal objective of this work was to study anterograde amnesia in several patients with TGA during the attack, with the aid of a prospectively designed protocol aimed at differentiating between selective disorders of encoding, storage, and retrieval of information in episodic memory. More specifically, our 
protocol should allow us to test the hypothesis that anterograde and retrograde amnesia result from distinct cognitive mechanisms, an opportunity otherwise difficult to test in permanent amnesia.

\section{Methods \\ SUBJECTS \\ Control subjects}

The control group consisted of 40 subjects aged between 45 and 75 (mean 57.7 (SD 9.1) years). To allow subsequent matching to prospectively recruited TGA patients, this control group was further subdivided into four subgroups of 10 subjects each according to age (two age ranges: 45-60 and 61-75 years) and educational level (for the young group: above or below 10 years of education; for the old group: above or below 8 years of education; the class boundary being different to lessen cohort effects).

\section{Patients}

Over a period of 1 year, we had the opportunity of examining eight patients suspected of having an episode of idiopathic TGA in the emergency department of the University Hospital of Caen. Six of them satisfied the operational criteria established by Caplan $^{19}$ and modified by Hodges and Warlow, ${ }^{20}$ but three had already entered the recovery stage when the neuropsychological tests were carried out. The protocol could be completed in its entirety during the acute phase by three patients. Their results are the basis of this study. There were two women (patient A and patient B), aged respectively 71 and 68 years, both with a good educational level and a man of 54 years, patient $\mathrm{C}$, with a low educational level.

NEUROPSYCHOLOGICAL PROTOCOL

The neuropsychological protocol was specially designed for this study. It is modular and can be applied in the form of short sequences interleaved with medical examinations.

\section{General cognitive assessment}

The general cognitive assessment included the following subtests: orientation in time and space, ${ }^{21}$ problem solving, and copying of geometric figures (taken from the cognitive assessment battery ${ }^{22}$ ), short term memory, assessed by the forward span, ${ }^{21}$ and semantic memory, assessed by means of categorical fluency (names of animals) and orthographical fluency (names beginning with the letter $\mathrm{p}$ ). In both cases the patient must provide a maximum number of names in 2 minutes. ${ }^{23} 24$

Assessment of episodic memory

Procedure-The battery for episodic memory was designed specifically to differentiate between encoding, storage, and retrieval, while accounting for the presence of massive anterograde amnesia during the tests. It consists of one list of 16 words belonging to 16 different semantic categories-presented one by one orally together with an instruction for memorisation-which the subject is required to reproduce explicitly. The words are concrete nouns of 5 to 8 letters, with a lexical frequency ranging from 100 to 10 000, selected from the Brulex lexical data bank. ${ }^{25}$ Our paradigm is derived from Grober and Buschke's procedure ${ }^{26}$ and uses the encoding depth principle of Tulving and Thompson, ${ }^{27}$ according to which the greater the depth of the processing of information the better it is recalled (generally semantic processing would be involved). To control the level of processing, the subject is first asked to process each word in depth by generating a sentence containing the word. Then, to ensure the actual carrying out of deep encoding, a task of immediate cued recall is given by providing the appropriate semantic category. This task is carried out every two words, the time elapsed between the presentation of the target word and the cued recall task thus remaining within the limits of working memory (appendix). Therefore, after processing for example, the first two words of the list (turnip, museum), the subject must recall each one of them in response to the presentation of their respective semantic cues (vegetable and public place respectively). This procedure is repeated throughout the whole list providing the score of immediate cued recall. If the subject fails in the immediate recall task, he or she is reminded of the word, again requested to make a sentence containing the target, and to recall it in response to its categorical cue. This procedure therefore ensures that even if the instruction to memorise is forgotten during the test, the items have been processed and therefore should leave a memory trace, if encoding is preserved. Immediately after the processing of the 16 words, retrieval is assessed according to both free recall and recognition. In the free recall procedure, the subject has to engage in a strategic search process which is not necessary in the recognition task because each target item is provided for him or her together with three distractors from the same semantic category the position of which was randomised.

ANALYSIS OF THE SCORE PROFILES

The battery produces three scores-namely, immediate cued recall, free recall, and recognition scores. The analysis of the score profiles makes it possible to infer the nature of the disturbance responsible for the anterograde amnesia. Thus, in the case of an encoding disturbance, patients will not be able to recall the target words in immediate cued recall ${ }^{28}$ and therefore, both free recall and recognition will be defective. In the case of a storage disturbance, despite adequate processing and preserved immediate cued recall in the encoding task, performances in free recall and recognition will be significantly affected. In fact, neither semantic processing nor recognition help at the stage of recall whenever encoding or storage are deficient. The comparison between free recall and recognition tasks is a standard method for showing specific disturbances in retrieval. ${ }^{26}$ Thus, where there is a selective retrieval disorder, performances should be clearly better in recognition than in free recall. This procedure should, therefore, make it possible to deduce whether the disorder is 
predominantly in the encoding, retrieval, or storage of information in the episodic memory.

\section{Execution of the test battery}

The tests were carried out in the following order: orientation in time and space, copying of geometric figures, memory span, episodic memory test, verbal fluency (categorical and orthographical), problem solving. The duration for the execution of the battery was about 1 hour.

Each patient was studied first during the acute stage of TGA, a second time on the next day, and a third time roughly a month later. The battery was also completed by the control group on two occasions at an interval of 1 month.

\section{ANALYSIS OF THE DATA}

Two subgroups were extracted from the population of control subjects and matched according to educational level and age: the first (mean 65.7 (SD 4.78)) with patient $\mathrm{A}$ and patient $\mathrm{B}$, and the second (mean 48.7 (SD 2.54)) with patient C.

The results obtained during the TGA episode and on the following day were compared with the results of the control subjects obtained at their first test session. The results obtained by the patients 1 month after the attack were compared with those from the control subjects' second test session. For each raw score, a $\mathrm{z}$ score was calculated and considered significant if it was $>1.83(\mathrm{p}<0.05$, one tailed).

\section{Results}

CONTROL SUBJECTS

Table 1 gives the results of the control subjects (first group matched with patient $\mathrm{A}$ and patient $B$ and the second group matched with patient C) for the battery of neuropsychological tests on two occasions at an interval of 1 month.

\section{PATIENTS}

Clinical findings

Patient A, a 71 year old right handed woman, without any medical history other than arterial hypertension (treated for several years with $\beta$

Table 1 Neuropsychological results of the control subjects on the two sessions: data shown are mean scores $(S D)$

\begin{tabular}{|c|c|c|c|c|}
\hline & \multicolumn{2}{|l|}{ Group $1^{\star}$} & \multicolumn{2}{|l|}{ Group $2 t$} \\
\hline & Session 1 & Session 2 & Session 1 & Session 2 \\
\hline \multicolumn{5}{|l|}{ Cognitive functions: } \\
\hline Orientation in time $(/ 5)$ & $4.90(0.32)$ & $5 \ddagger$ & $5 \ddagger$ & $5 \ddagger$ \\
\hline Problem solving (/12) & $11.70(0.67)$ & $11.70(0.48)$ & $11.80(0.42)$ & $12 \ddagger$ \\
\hline \multicolumn{5}{|l|}{ Copying of geometric } \\
\hline figures (/12) & $11.40(1.07)$ & $11.50(0.84)$ & $11.60(0.70)$ & $11.70(0.48)$ \\
\hline Forward memory span & $5.90(0.87)$ & $5.90(0.74)$ & $6.40(1.35)$ & $5.90(0.74)$ \\
\hline \multicolumn{5}{|l|}{ Categorical fluency } \\
\hline Correct responses & $33.90(5.15)$ & $35.50(5.10)$ & $34.80(7.69)$ & $32.90(7.80)$ \\
\hline Perseverative errors & $0.90(0.99)$ & $0.8 \quad(0.79)$ & $0.2(0.42)$ & $0.3(0.48)$ \\
\hline \multicolumn{5}{|l|}{ Letter fluency } \\
\hline Correct responses & $(5.25)$ & $25.30(4.11)$ & $22.20(6.49)$ & $22.10(5.47)$ \\
\hline Perseverative errors & $0.2(0.42)$ & $0.4 \quad(0.52)$ & $0.3(0.67)$ & $0.3(0.48)$ \\
\hline \multicolumn{5}{|l|}{ Episodic memory: } \\
\hline Immediate cued recall(/16) & $16 \ddagger$ & $16 \ddagger$ & $16 \ddagger$ & $16 \ddagger$ \\
\hline Free recall $(/ 16)$ & $7.90(1.37)$ & $8.40(2.59)$ & $9.50(1.35)$ & $9.7 \quad(2.06)$ \\
\hline Recognition (/16) & $15.70(0.67)$ & $16 \ddagger$ & $15.70(0.67)$ & $16 \ddagger$ \\
\hline
\end{tabular}

${ }^{\star}$ Group 1 is matched with patients A and B.

tGroup 2 is matched with patient $C$.

$\ddagger \mathrm{SD}=0$. blockers and diuretics) and hypercholesterolaemia, was at a swimming pool with a friend on 11 June 1996. At around $300 \mathrm{pm}$, as she came out of the pool, having swum 300 metres, her friend noticed that her behaviour was unusual; she asked repetitive questions about her presence at the pool, the day, the hour, and the date. Accompanied by her friend, patient $\mathrm{A}$ went to her daughter, a pharmaceutical chemist. Her daughter called the family doctor, who noted an arterial blood pressure of $170 / 90$. She was referred to the University Hospital of Caen and arrived there accompanied by her husband at around $600 \mathrm{pm}$. Neurological examination did not show any focal abnormality other than that of memory. An ECG showed no significant abnormality and routine blood testing was normal. Brain CT was normal. Neuropsychological testing started at $800 \mathrm{pm}$ while the patient was still deeply amnesic. The psychologist noted memory impairment until she left at $1045 \mathrm{pm}$. Retrograde amnesia was estimated at about 2 months. Patient A had forgotten the death of a close friend and was astonished when her husband confirmed that the friend had in fact died 2 months previously. The next day the patient showed no signs of memory disturbance apart from a lacunar amnesia concerning the day of the attack. An ultrasound investigation of the cervical arteries with B-mode scanning and transcranial Doppler ultrasonography were normal.

Patient B, a 68 year old right handed woman, without any medical history apart from previous arterial hypertension treated with $\beta$ blockers and diuretics, was at home with her husband and grandchildren on 4 December 1996. At around 1130 am she started repetitive questioning concerning the date and her short term plans. When seen at the emergency ward at $1245 \mathrm{pm}$, neurological examination disclosed disorientation for time but not for space, anterograde amnesia with persistence of repetitive questioning but normal awareness, and no other focal neurological abnormality. Cardiac examination was normal, ECG showed a sinusal rhythm, and routine blood testing was normal. Brain CT showed mild diffuse cortical atrophy and one lacunar hypodensity on the lateral part of each putamen, as well as grade I leukoaraiosis. Neuropsychological examination started around $500 \mathrm{pm}$. At this time patient B still had both severe anterograde and retrograde amnesia covering the previous weekend. She knew that some of her grandchildren were at her house but not which of them; she did not remember her activities of the previous day and seemed anxious and very intrigued at the presence of a necklace around her neck. This indicated that she had had to leave home but was not connected with any specific memory. She had forgotten that she took her grandchildren back to their house the same day. Around 700 pm the recovery phase began but memory disturbances persisted for the entire evening. The next day the patient presented no memory impairment apart from lacunar amnesia going back to the final hours of the day before. An EEG and a Doppler examination of the supra- 
aortic and intracranial vessels, as well as carotid ultrasonography, were normal. T2 weighted MRI showed no additional lesions.

Patient C, a 54 year old right handed man without any medical history other than migraine without aura, was at home on 7 November 1996. At about 1030 am, after sexual intercourse, he questioned his wife repetitively, asking her why he had to change his clothes (he was invited to lunch). He had forgotten what he had done during the morning, particularly a gym session, which had finished at $1000 \mathrm{am}$. His family doctor reported memory disturbances without any associated neurological disorder. On his arrival at the University Hospital of Caen at $1130 \mathrm{am}$, accompanied by his wife and son, a neurological examination showed normal awareness, mnesic disturbance, disorientation in time without disorientation in space, and no other abnormality. Brain CT was normal and ECG and routine blood testing showed no special features. The neuropsychological examination began around $130 \mathrm{pm}$ when the patient still had severe anterograde amnesia and total disorientation in time. The retrograde amnesia seemed to concern several months; in particular he had forgotten a trip to Canada in August 1996. The next day the patient had no memory disturbance other than lacunar amnesia concerning the day of the attack (lasting from 1000 am until the evening). During his stay in hospital, arterial hypertension with a maximum of $250 \mathrm{~mm} \mathrm{Hg}$ was found. Doppler examination of supraaortic and transcranial vessels was normal, and the EEG only showed slowed bifrontotemporal reactivity during hyperventilation.

\section{Neuropsychological scores during TGA}

Table 2 gives the three patients' scores for the battery of neuropsychological tests during the attack. Orientation in time was significantly disturbed in all three patients. Reasoning (assessed by problem solving), visuoconstructive abilities (copying geometric figures), and short term memory (verbal forward span) were preserved in all cases. The number of correct responses in both verbal fluency tasks was significantly low for patient $\mathrm{A}$ and on the borders of significance for patient C. These two

Table 2 Neuropsychological results during TGA: data shown are raw scores (and corresponding z scores)

\begin{tabular}{lccc}
\hline & Patient $A$ & Patient $B$ & Patient $C$ \\
\hline Cognitive functions: & & & \\
$\quad$ Orientation in time $(/ 5)$ & $3(-5.93)^{\star \star \star}$ & $4(-2.82)^{\star \star}$ & $3 \dagger$ \\
Problem solving $(/ 12)$ & $12(+0.25)$ & $\mathrm{ND}$ & $12(+0.47)$ \\
Copying of geometric figures $(/ 12)$ & $12(+0.35)$ & $12(+0.35)$ & $12(+0.57)$ \\
$\quad$ Forward memory span & $7(+1.26)$ & $8(+2.41)$ & $6(-0.29)$ \\
Categorical fluency: & $22(-2.31)^{\star}$ & $37(+0.60)$ & $21(-1.79)$ \\
$\quad$ Correct responses & $1(-0.10)$ & $26(-25.35)^{\star \star \star}$ & $2(-4.28)^{\star \star \star}$ \\
$\quad$ Perseverative errors & $14(-2.09)^{\star}$ & $40(+2.85)$ & $12(-1.57)$ \\
Letter fluency: & $3(-6.67)^{\star \star \star}$ & $7(-16.19)^{\star \star \star}$ & $2(-2.53)^{\star \star}$ \\
$\quad$ Correct responses & & & \\
$\quad$ Perseverative errors & $8 \dagger$ & $16 \ddagger$ & $15 \dagger$ \\
Episodic memory: & $1(-5.03)^{\star \star \star}$ & $3(-3.57)^{\star \star}$ & $2(-5.55)^{\star \star \star}$ \\
Immediate cued recall (/16) & $1(-21.94)^{\star \star \star}$ & $6(-14.47)^{\star \star \star}$ & $10(-8.50)^{\star \star \star}$ \\
Free recall $(/ 16)$ & & & \\
Recognition $(/ 16)$ & & &
\end{tabular}

${ }^{\star} \mathrm{p}<0.05 ;{ }^{\star \star} \mathrm{p}<0.01 ;{ }^{\star \star \star}{ }^{\star \star} \mathrm{p}<0.001$.

†Impaired performance (z score not assessable as $\mathrm{SD}=0$ in controls).

$\mathrm{ND}=$ not done.

$\ddagger$ Normal performance (z score not assessable as $\mathrm{SD}=0$ in controls). patients produced several perseverative errors both in categorical and letter fluency. These results stand in contrast with those of patient B who, despite a normal number of correct responses, produced a great number of perseverative errors in both fluency tasks.

For the episodic memory test, the three patients carried out the semantic processing task correctly (making a sentence). The sentences were produced quickly and without error but patient A was able to recall only eight of 16 words on the first trial and the ensuing eight on the second trial in the immediate cued recall. Her performances were also significantly impaired in both free recall and recognition. Taken together for patient A, these results suggest an encoding impairment. For the other two patients, immediate cued recall was either strictly normal (patient B) or just below maximal score (patient C) indicating effective encoding, which contrasted with low free recall and recognition scores, suggesting a storage impairment even if recognition scores were higher than free recall scores.

Neuropsychological results the day after TGA

Table 3 shows the three patients' results for the battery of neuropsychological tests the day after the transient global amnesia. All the results were back to normal for patient $A$ and patient B (however, patient A did not do the verbal fluency test). Patient C still had a reduced score in categorical verbal fluency (already down, although not significantly so, the previous day).

Neuropsychological and neurological follow up

The battery of neuropsychological tests carried out about 1 month after the attack gave normal results for all the patients (table 3). A medical and psychological follow up of these patients several months after the attack showed that all were in good health, had not had any new neurological episode, and did not complain of any memory difficulties.

\section{Discussion}

We have carried out what is to the best of our knowledge the first prospective study especially designed to examine the cognitive characteristics of anterograde amnesia in idiopathic TGA using a procedure derived from Grober and Buschke. ${ }^{26}$ The results obtained favour the idea of a disturbance in the encoding in one patient (A) and the storage of information for the other two patients (B and $\mathrm{C}$ ).

CLINICAL CONSIDERATIONS

Each of the three patients satisfied the operational criteria for transient global amnesia proposed by Caplan ${ }^{19}$ and modified by Hodges and Warlow. ${ }^{20}$ Epilepsy, stroke, and head injury were ruled out by means of a reasonably extensive investigation. The follow up several months after the episode showed them to be in good health with no complaints of memory disturbance. During the attack, anterograde amnesia was severe and was associated with disorientation in time, contrasting with main- 
Table 3 Neuropsychological results the day after the TGA episode and at follow up: data shown are raw scores (and corresponding z scores)

\begin{tabular}{|c|c|c|c|c|c|c|}
\hline & \multicolumn{2}{|l|}{ Patient $A$} & \multicolumn{2}{|l|}{ Patient B } & \multicolumn{2}{|l|}{ Patient $C$} \\
\hline & Day after & Follow up & Day after & Follow up & Day after & Follow up \\
\hline \multicolumn{7}{|l|}{ Cognitive functions: } \\
\hline Orientation in time $(/ 5)$ & $5(+0.31)$ & $5+$ & $5(+0.31)$ & $5+$ & $5+$ & $5+$ \\
\hline Problem solving $(/ 12)$ & ND & $12(+0.62)$ & $12(+0.47)$ & $12(+0,62)$ & $12(+0.47)$ & $12 \dagger$ \\
\hline Copying of geometric figures (/12) & $12(+0.56)$ & $12(+0.59)$ & $12(+0.56)$ & $11(-0,59)$ & $12(+0.57)$ & $12(+0.62)$ \\
\hline Forward memory span & $7(+1.26)$ & $7(+1.49)$ & $7(+1.26)$ & $6(0,13)$ & $6(-0.29)$ & $6(+0.13)$ \\
\hline \multicolumn{7}{|l|}{ Categorical fluency: } \\
\hline Correct responses & ND & $46(+2.06)$ & $49(+2.93)$ & $57(+4,21)$ & $16(-2.47)^{\star}$ & $25(-1.01)$ \\
\hline Perseverative errors & & $0(+1.01)$ & $2(-1.11)$ & $0(+1.01)$ & $1(-1.93)^{\star}$ & $0(+062)$ \\
\hline \multicolumn{7}{|l|}{ Letter fluency: } \\
\hline Correct responses & ND & $22(-0.8)$ & $44(+3.62)$ & $44(+4.55)$ & $16(-0.95)$ & $24(+0.35)$ \\
\hline Perseverative errors & & $0(+0.77)$ & $0(+0.48)$ & $0(+0.77)$ & $0(+0.44)$ & $0(+0.62)$ \\
\hline \multicolumn{7}{|l|}{ Episodic memory: } \\
\hline Immediate cued recall $(/ 16)$ & $16 \dagger$ & $16+$ & $16 \dagger$ & $16 \dagger$ & $16 \dagger$ & $16 \dagger$ \\
\hline Free recall $(/ 16)$ & $7(-0.65)$ & $11(+1.00)$ & $12(+2.99)$ & $10(+0.62)$ & $10(+0.37)$ & $9(-0.34)$ \\
\hline Recognition (/16) & $16(+0.45)$ & $16 t$ & $16(+0.45)$ & $16+$ & $16(+0.45)$ & $16 t$ \\
\hline
\end{tabular}

${ }^{\star} \mathrm{p}<0.05$.

†Normal performance (see table 2).

$\mathrm{ND}=$ not done.

tained reasoning, visuoconstructive, and short term memory (at least the phonological loop, which is principally involved in forward digit span) abilities.

Our three patients had disturbed verbal fluency both in number of correct responses (during the acute stage for patient $\mathrm{A}$, or the next day for patient $\mathrm{C}$, but in $\mathrm{C}$, the score obtained during the episode was close to the pathological threshold) and in number of perseverative errors. At follow up, all these scores became normal. Impaired verbal fluency (either categorical or letter) has previously been reported during ${ }^{9}{ }^{16}$ or after $^{30}$ a TGA episode. A low correct response score has been interpreted as reflecting impaired strategy of retrieval from semantic memory. ${ }^{9}$ The perseverative errors are in keeping with the severe episodic memory deficit rather than with an impairment of semantic memory, the preservation of which has previously been demonstrated during TGA. ${ }^{5}$ In the same way, the normal ability of our patients to generate sentences from words in the episodic memory test argues in favour of preserved semantic memory.

The day after the episode, the performances in episodic memory tests were normal in all patients. Only few investigators have reported the persistence of a clinically undetectable episodic memory impairment on the day after TGA. ${ }^{31} 32$

\section{ANTEROGRADE AMNESIA}

The principal objective of this prospective study was to analyse the mechanisms of the anterograde amnesia that characterises the acute phase of TGA. A protocol was purposely designed to allow the distinction between disturbances in encoding, storage, or retrieval of information in the episodic memory. This protocol, based on verbal material, controlled for both the type of semantic processing used at the encoding stage (deep encoding) and the type of processing used at retrieval (free recall or recognition). Our three patients presented two distinct impairment patterns. Whereas all three patients showed accurate semantic processing of the items, as established by good sentence production, only two of them (patient $B$ and patient $C$ ) performed correctly at the immediate cued recall task. The last patient (A) gave only half of the items on the first trial (and the remaining on the second trial). This may reflect difficulties in simultaneously carrying out two tasks (maintaining a word and making a sentence with another word), an ability that depends on the central executive component of working memory. ${ }^{33}$ This may result in defective elaborate encoding that requires items not only being deeply processed, but also meaningfully associated with knowledge already stored in long term memory. ${ }^{34}$ Nevertheless, the other components of working memory (including the phonological loop) would be intact; hence the normal forward digit span in patient A. This encoding deficit in turn would readily explain patient A's poor performances in free recall and recognition (see $\mathrm{s}^{35-37}$ for discussion in permanent amnesia)

The two other patients did encode the verbal material, but were impaired at both free recall and recognition, although recognition scores were better preserved. This pattern would not fit the hypothesis of a predominance of a retrieval problem. The better performance in recognition relative to recall tasks may reflect implicit processes. $^{38}$ which are preserved in TGA. ${ }^{9}$ All things considered, the lack of efficacy of deep semantic processing and recognition does suggest a preferential storage disturbance $\left(\mathrm{se}^{39}\right.$ for a discussion of permanent amnesia). The fact that the performance of patient $\mathrm{C}$ at the immediate cued recall was just below the maximal score (table 2) could suggest a participation of some encoding impairment as well.

As has been shown recently by Kapur et al, ${ }^{10}$ even massive anterograde amnesia does not prevent encoding, storage, and retrieval of new information when implicit tasks are involved. These authors showed intact priming effects not only during the TGA episode, ${ }^{7-9}$ but also 7 days later for information encoded during the episode. If results of Kapur et al and ours are considered together, it emerges that the disturbance in either encoding or storage during TGA is restricted to explicit memory. Future studies examining the speed of forgetting 
should be able to clarify whether the disturbance in consolidation manifests itself during the passage from working memory to episodic memory or within episodic memory itself.

\section{RETROGRADE $V$ ANTEROGRADE AMNESIA}

In our patients, retrograde amnesia was not as systematically assessed as anterograde amnesia because of methodological restrictions due to the brevity of the attack. However, certain clinical elements indicate that retrograde amnesia did exist in all, and was even extensive in two of our three cases. In addition, several prospective studies have assessed retrograde amnesia, emphasising its extensiveness and the fact that it essentially involves episodic memory. ${ }^{11}{ }^{13}$ As TGA occurs suddenly and as the memories preceding the episode (but inaccessible during it) may be recalled when the episode is over, the most likely mechanism to explain retrograde amnesia in TGA is impairment in the retrieval of episodic information. As our results indicate that anterograde amnesia in TGA is due to either or both an encoding or a storage deficiency, it follows that in TGA anterograde and retrograde amnesia are caused by different mechanisms. Previously, Hodges and Ward ${ }^{3}$ suggested this hypothesis, but on the basis of clinical arguments only-such as the severity of anterograde amnesia as compared with a more erratic nature of retrograde amnesia. This variability in the extent of retrograde amnesia from patient to patient could reflect the commensurate variability in the time the evaluation is carried out within the course of the episode. Indeed, there exists a point in time in the recovery phase when anterograde amnesia is still significant but retrograde amnesia has already almost vanished. ${ }^{40} \mathrm{~A}$ difference in the intensity of the two types of amnesia has also been emphasised in permanent amnesic syndromes, ${ }^{41}$ the clearest dissociation having been found in focal retrograde amnesia. ${ }^{42} 43$ However, these studies have only pointed out quantitative differences in the duration of retrograde versus anterograde amnesia, without going into the mechanisms involved. The present work provides experimental evidence for dissociated impairment within episodic memory processes and gives further support to the idea of functional independence of these processes. Such a dissociation in the mechanisms of anterograde and retrograde amnesia has been extensively discussed by Evans et $a l^{44}$ in a case report of focal retrograde amnesia after primary cerebral vasculitis. The authors qualified this dissociation and discussed the pattern of impairment of their patient in the framework of connectionist models of memory and amnesia. ${ }^{45}{ }^{46}$ Further prospective studies in TGA are required, using protocols specifically designed to assess the fine grained mechanisms of both anterograde amnesia and retrograde amnesia, notably about the temporal gradient.

We thank the physicians of the Emergency Department of the University Hospital of Caen, and especially Dr Bruno Le Chevalier, for help in this project.
Appendix: Episodic memory task

List of 16 words (roman case) and corresponding cues (italics, the semantic category provided by the examinator in the immediate cued recall task that takes place every two words)

1 navet (turnip)

2 musée (museum)

1 légume (vegetable)

2 lieu public (public place)

3 tambour (drum)

4 buffet (sideboard)

3 instrument de musique (instrument)

4 meuble (piece of furniture)

5 raisin (grapes)

6 muguet (lily)

5 fruit (fruit)

6 fleur (flower)

7 sapin (fir)

8 renard (fox)

arbre (tree)

8 mammifère (mammal)

9 pyjama (pyjamas)

10 ventre (stomach)

9 vêtement (clothes)

10 partie du corps (body part)

11 couteau (knife)

12 dentiste (dentist)

11 ustensile de cuisine (kitchen utensil)

12 profession (profession)

13 hache (axe)

14 voilier (sailing ship)

13 outil (tool)

14 transport maritime (shipping)

15 colombe (dove)

16 sardine (sardine)

15 oiseau (bird)

16 poisson (fish)

1 Fisher CM, Adams KD. Transient global amnesia. Transactions of the American Neurology Association 1958;83:143-6.

Fisher CM, Adams RD. Transient global amnesia. Acta Neurol Scand 1964;40(suppl.9):1-83.

3 Hodges JR, Ward CD. Observations during transient global amnesia: a behavioural and neuropsychological study of amnesia: a behavioural and neurop
five cases. Brain 1989;112:595-620.

4 Regard M, Landis T. Transient global amnesia: neuropsychological dysfunction during attack and recovery in two pure cases. $\mathcal{F}$ Neurol Neurosurg Psychiatry 1984;47:66872

5 Hodges JR. Semantic memory and frontal executive function during transient global amnesia. $\mathcal{F}$ Neurol Neurosurg Psychiatry 1994;57:605-8.

6 Goldenberg G. Transient global amnesia. In: Baddeley AD, Wilson BA, Watts FN, eds. Handbook of memory disorders. Chichester: J Wiley, 1995:109-33.

7 Goldenberg G, Podreka I, Pfaffelmeyer N, et al. Transient ischemia in transient global amnesia: a SPECT study. Neurology 1991;41:1748-52.

8 Kazui H, Tanabe H, Ikeda Y, et al. Memory and cerebral blood flow in cases of transient global amnesia during and after the attack. Behavioural Neurol 1995;8:93-101.

9 Eustache F, Desgranges B, Petit-Taboué MC, et al. Transient global amnesia: implicit/explicit memory disso-
Tracher ciation, and PET assessment of brain perfusion and oxygen metabolism in the acute stage. F Neurol Neurosurg Psychiatry 1997;63:357-67.

10 Kapur N, Abbott P, Footitt D, et al. Long-term perceptual priming in transient global amnesia. Brain Cogn 1996;31: 63-74.

11 Kritchevsky M, Squire LR, Zouzounis JA. Transient global amnesia: characterization of anterograde and retrograde amnesia. Neurology 1988;38:213-19.

12 Kritchevsky M, Squire LR. Transient global amnesia: evidence for extensive, temporally graded retrograde amnesia. Neurology 1989;39:218-31.

13 Evans J, Wilson B, Wraight EP, et al. Neuropsychological and SPECT scan findings during and after transient global amnesia: evidence for the differential impairment of remote episodic memory. 7 Neurol Neurosurg Psychiatry 1993;56: 1227-30.

14 Baddeley A. The psychology of memory. In: A Baddeley, BA Wilson, FN Watts, eds. Handbook of memory disorders. Chichester: J Wiley, 1995:3-25.

15 Gordon B, Marin OSM. Transient global amnesia: an Gordon B, Marin OSM. Transient global amnesia: an extensive case

16 Caffara P, Moretti G, Mazzucchi A, et al. Neuropsychological testing during a transient global amnesia episode and its follow up. Acta Neurol Scand 1981;63:44-50.

17 Härting C, Markovitsch J. Different degrees of impairment in anterograde/retrograde memory and recall/recognition performance in transient amnesic case. Neurocase 1996;2: 45-9.

18 Baron JC, Petit-Taboué MC, Le Doze F, et al. Right frontal cortex hypometabolism in transient global amnesia. A PET study. Brain 1994;117:545-52.

19 Caplan LR. Transient global amnesia. In: Fredericks JAM, ed. Handbook of clinical neurology. Amsterdam: Elsevier, 1985:205-18.

20 Hodges JR, Warlow CP. Syndromes of transient global amnesia: towards a classification: a study of 153 cases. $\mathcal{F}$ Neurol Neurosurg Psychiatry 1990;53:834-43.

21 Wechsler D. Wechsler memory scale. Paris: Psychological Corporation, Editions du Centre de Psychologie Appliquée, 1969. 
22 Signoret JL, Allard M, Benoit N, et al, eds. Evaluation des troubles de mémoire et des désordres cognitifs associés, BEC 96. troubles de mémoire et des désordres

23 Cardebat D, Doyon B, Puel M, et al. Evocation lexicale formelle et sémantique chez des sujets normaux: performances et dynamiques de production en fonction du sexe, de l'âge et du niveau d'étude. Acta Neurol Belg 1990;90:20717 .

24 Desgranges B, Eustache F, Rioux P, et al. Memory disorders in Alzheimer's disease and the organization of human memory. Cortex 1996;32:387-412.

25 Content A, Mousty P, Radeau M. Brulex: une base de données lexicales informatisée pour le français écrit et parlé. L'anné Psychologique 1990;90:551-66.

26 Grober E, Buschke H. Genuine memory deficits in dementia. Dev Neuropsychol 1987;3:13-36.

27 Tulving E, Thompson DM. Encoding specificity and retrieval processes in episodic memory. Psychol Rev retrieval processes

28 Mayes AR, Downes JJ, Shoqeirat M, et al. Encoding ability is preserved in amnesia: evidence from a direct test of is preserved in amnesia: evidence from a
encoding. Neuropsychologia 1993;31:745-59.

29 Lin KN, Liu RS, Yeh TP, et al. Posterior ischemia during an attack of transient global amnesia. Stroke 1993;24:1093-5.

30 Stilhard G, Landis T, Schiess R, et al. Bitemporal hypoperfusion in transient global amnesia: 99m-tc-HMPAO SPECT and neuropsychological findings during and after an attack. F Neurol Neurosurg Psychiatry 1990;53:339-42.

31 Mazzucchi A, Moretti G, Caffara P, et al. Neuropsychological functions in the follow-up of transient global amnesia. Brain 1980;103:161-78.

32 Hodges JR, Oxbury SM. Persistent memory impairment following transient global amnesia. F Clin Exp Neuropsychol 1990;12:904-20.

33 Baddeley A. The fractionation of working memory. Proc Natl Acad Sci USA 1996;93:13468-72.
34 Schacter DL. Searching for memory. The brain, the mind, and the past. New York: Basic Books, 1996.

35 Cermak LS. A positive approach to viewing processing deficit theories of amnesia. Memory 1997;5:89-99.

36 Keane MM, Gabrieli JDE, Monti LA, et al. Intact and impaired conceptual memory processes in amnesia. Neuropsychology 1997;11:59-69.

37 Mayes AR, Downes JJ. What do theories of functional deficit(s) underlying amnesia have to explain. Memory 1997;5: 3-37.

38 Mandler G, Overson Hamson C, Dorfman J Tests of dual process theory: word priming and recognition. $O \mathcal{F}$ Exp Psychol A 1990;42:713-39.

39 Cermak LS, O'Connor M. The anterograde and retrograde retrieval ability of patient with amnesia due to encephalitis. Neuropsychologia 1983;21:213-34

40 Kazui H, Tanabe H, Ikeda M, et al. Retrograde amnesia during transient global amnesia. Neurocase 1996;2:127-33.

41 Schmidtke K, Vollmer H. Retrograde amnesia: a study of its relation to anterograde amnesia and semantic memory deficits. Neuropsychologia 1997;35:505-18.

42 Kapur N. Focal retrograde amnesias in neurological disease: a critical review. Cortex 1993;29:217-34.

43 O'Connor M, Butters N, Militosis P, et al. The dissociation of retrograde and anterograde amnesia in a patient with of retrograde and anterograde amnesia in a patient with
herpes encephalitis. F Clin Exp Neuropsychol $1992 ; \mathbf{1 4}: 159-$ 78 .

44 Evans JJ, Breen EK, Antoun N, et al. Focal retrograde amnesia for autobiographical events following cerebral vasculitis: a connectionist account. Neurocase 1996;2:1-11.

45 Murre JMJ. TraceLink: a model of amnesia and consolidation of memory. Hippocampus 1996;6:675-84.

46 Murre JMJ. Implicit and explicit memory in amnesia: some explanations and predictions by the TraceLink model. Memory 1997,5:213-32. 